\title{
Dietary Soy Supplement on Fibromyalgia Symptoms: A Randomized, Double-Blind, Placebo-Controlled, Early Phase Trial
}

\author{
Dietlind L. Wahner-Roedler, ${ }^{1}$ Jeffrey M. Thompson, ${ }^{2}$ Connie A. Luedtke, ${ }^{3}$ Susan M. King, ${ }^{3}$ \\ Stephen S. Cha, ${ }^{4}$ Peter L. Elkin, ${ }^{1}$ Barbara K. Bruce, ${ }^{5}$ Cynthia O. Townsend, ${ }^{5}$ \\ Jody R. Bergeson, ${ }^{3}$ Andrea L. Eickhoff, ${ }^{3}$ Laura L. Loehrer, ${ }^{1}$ Amit Sood, ${ }^{1}$ and Brent A. Bauer ${ }^{1}$
}

${ }^{1}$ Division of General Internal Medicine, Mayo Clinic, Rochester, MN, USA

${ }^{2}$ The Fibromyalgia Treatment Program, Mayo Clinic, Rochester, MN, USA

${ }^{3}$ The Fibromyalgia Treatment/Rehabilitation Center, Mayo Clinic, Rochester, MN, USA

${ }^{4}$ The Division of Biostatistics, Mayo Clinic, Rochester, MN, USA

${ }^{5}$ The Division of Tertiary Psychiatry and Psychology, Mayo Clinic, Rochester, MN, USA

Correspondence should be addressed to Dietlind L. Wahner-Roedler, wahnerroedler.dietlind@mayo.edu

Received 25 November 2008; Accepted 28 May 2009

Copyright (C) 2011 Dietlind L. Wahner-Roedler et al. This is an open access article distributed under the Creative Commons Attribution License, which permits unrestricted use, distribution, and reproduction in any medium, provided the original work is properly cited.

\begin{abstract}
Most patients with fibromyalgia use complementary and alternative medicine (CAM). Properly designed controlled trials are necessary to assess the effectiveness of these practices. This study was a randomized, double-blind, placebo-controlled, early phase trial. Fifty patients seen at a fibromyalgia outpatient treatment program were randomly assigned to a daily soy or placebo (casein) shake. Outcome measures were scores of the Fibromyalgia Impact Questionnaire (FIQ) and the Center for Epidemiologic Studies Depression Scale (CES-D) at baseline and after 6 weeks of intervention. Analysis was with standard statistics based on the null hypothesis, and separation test for early phase CAM comparative trials. Twenty-eight patients completed the study. Use of standard statistics with intent-to-treat analysis showed that total FIQ scores decreased by $14 \%$ in the soy group $(P=.02)$ and by $18 \%$ in the placebo group $(P<.001)$. The difference in change in scores between the groups was not significant $(P=.16)$. With the same analysis, CES-D scores decreased in the soy group by $16 \%(P=.004)$ and in the placebo group by $15 \%(P=.05)$. The change in scores was similar in the groups $(P=.83)$. Results of statistical analysis using the separation test and intent-to-treat analysis revealed no benefit of soy compared with placebo. Shakes that contain soy and shakes that contain casein, when combined with a multidisciplinary fibromyalgia treatment program, provide a decrease in fibromyalgia symptoms. Separation between the effects of soy and casein (control) shakes did not favor the intervention. Therefore, large-sample studies using soy for patients with fibromyalgia are probably not indicated.
\end{abstract}

\section{Introduction}

Fibromyalgia syndrome (FMS) is a chronic, generalized pain syndrome that affects the musculoskeletal system [1]. This syndrome is typically diagnosed in patients who experience generalized musculoskeletal pain and have excessive tenderness in at least 11 of 18 specific points [2]. Although the primary cause of FMS is unclear, a growing body of evidence indicates that the widespread pain associated with this syndrome is due to abnormalities in the central nervous system. Therefore, drug therapy for FMS is most often aimed at the central nervous system and includes tricyclic antidepressants, selective serotonin reuptake inhibitors, dual serotonin and norepinephrine reuptake inhibitors, analgesics and anticonvulsants [1].

In addition to medical therapies, complementary and alternative medicine (CAM) therapies have been used to treat FMS symptoms [3]. Overall, $>50 \%$ of patients with rheumatologic conditions, including FMS, use CAM therapies [4-6]. Wahner-Roedler et al. [6] reported that 98\% of patients who are referred to a fibromyalgia treatment program may have used some form of CAM within the 
last 6 months. The types of CAM therapies used to reduce FMS symptoms include massage, meditation, acupuncture, hypnotherapy and dietary supplementation. Few CAM interventions have been adequately tested in controlled clinical trials. In a systematic review published in 2003, Holdcraft et al. [3] concluded that the strongest evidence for efficacy in FMS exists for acupuncture. Magnesium use, S-adenosyl-Lmethionine (SAMe) use, and massage therapy have moderate evidence; chlorella use, relaxation, biofeedback, magnet therapies, homeopathy, botanical oils, balneotherapy and use of anthocyanidins have limited evidence. In a 2004 published summary of CAM trial data, Ernst [7] concluded that acupuncture and spinal manipulations have shown a significant promise in the treatment of FMS. No dietary supplement has conclusive evidence of efficacy in the treatment of FMS symptoms.

Soy is a widely used dietary supplement that has not been previously tested for treating FMS. On the basis of studies indicating that dietary soy relieves neuropathic pain in animals [8-11] and reduces pain and improves range of motion of the knee joints in humans with osteoarthritis [12], we hypothesized that soy consumption might improve FMS symptoms. The aim of our study was to evaluate whether dietary soy supplement can improve symptoms in patients with FMS participating in a 1.5-day multidisciplinary fibromyalgia treatment program, as measured by the Fibromyalgia Impact Questionnaire (FIQ) and the Center for Epidemiologic Studies Depression Scale (CES-D).

\section{Subjects and Methods}

This study was approved by the Mayo Clinic Institutional Review Board and registered as NCT00279942 in Clinical Trials.gov.

Patients presenting to the Mayo Fibromyalgia Treatment Program between May 2006 and August 2006 were invited to participate in this trial. Exclusion criteria included diagnoses of bipolar disorder, schizophrenia, dementia, diabetes mellitus and inflammatory bowel disease; allergy to soy or other study product ingredients; pregnancy; and consumption of soy products within the past 30 days. Among patients seen in our Fibromyalgia Treatment Program between May 2006 and August 2006, 117 met the study inclusion criteria and were invited to participate in the study (Figure 1). Of these, 67 patients declined to participate, and the remaining 50 patients were randomly assigned to either soy supplement or placebo.

2.1. The Mayo Fibromyalgia Treatment Program. The Mayo Fibromyalgia Treatment Program is a 1.5 day, multidisciplinary outpatient program staffed by physicians from the Department of Physical Medicine and Rehabilitation and the Department of Rheumatology. Access to this program is limited to patients with a presumed diagnosis of FMS who are referred by Mayo Clinic physicians. The patients undergo an initial evaluation by a registered nurse specifically trained in rheumatologic disorders, with the collaboration of a physician to confirm the diagnosis. Only patients with a

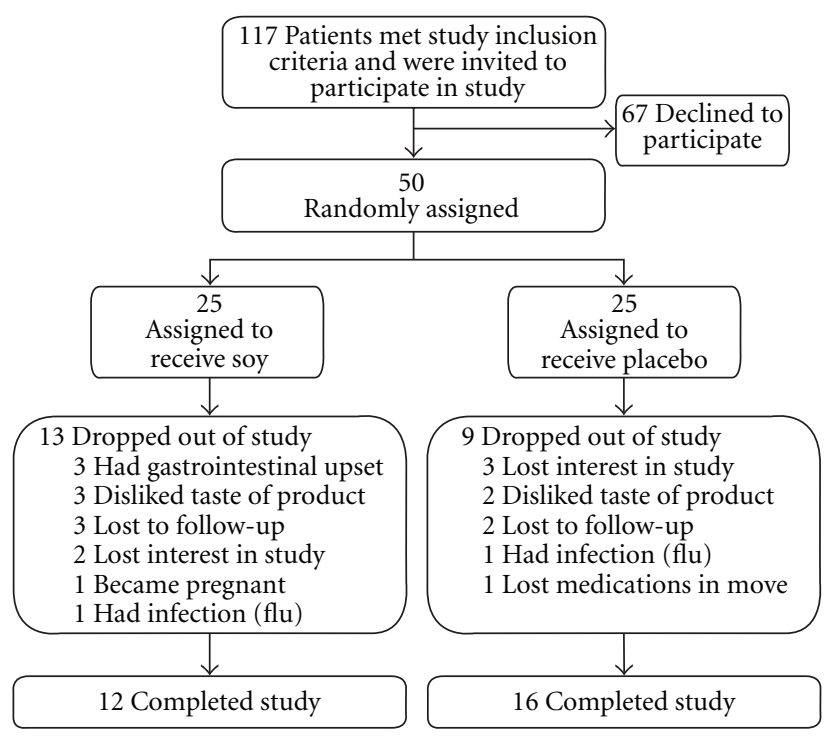

Figure 1: Flow chart of patients in the placebo-controlled soy supplement trial.

confirmed diagnosis of FMS as defined by the American College of Rheumatology in 1990-namely, widespread musculoskeletal pain of at least 3 months' duration and excessive tenderness in at least 11 of 18 predefined anatomical sites [2] - are enrolled in the program. Components of the program are carried out by a core group of team members that includes registered nurses, rheumatologists, psychiatrists, occupational therapists, physical therapists and ancillary staff. The mission of the fibromyalgia treatment program is to improve patients' physical and mental health functioning, impart evidence-based information and create a standardized treatment approach. Further details about this multidisciplinary program have been described [13].

2.2. Study Design. We conducted a randomized, doubleblind, placebo-controlled, early phase trial. Participants were randomly assigned to either soy supplement or placebo when they started the fibromyalgia treatment program. An informed consent was obtained from all participants. The participants completed the FIQ and the CES-D at baseline and were randomly assigned to either soy supplement or placebo taken once a day for 6 weeks. They were asked to collect wrappers of the product and to send them by mail in a provided envelope addressed to Mayo Clinic, together with another set of the completed FIQ and CES-D forms, at the end of the 6 weeks. The study coordinator, for whom the assignment of patients was blinded, called the participants weekly to inquire about product tolerance and compliance.

2.3. Products. The treatment was a soy shake (provided by Physicians Pharmaceuticals, Inc, Kernersville, NC, USA) that contained $20 \mathrm{~g}$ of soy protein and $160 \mathrm{mg}$ of soy isoflavone. The placebo was a shake that contained $20 \mathrm{~g}$ of milk-based protein (casein) and no isoflavone (provided by Physicians 
Pharmaceuticals, Inc). Flavoring, sweetening and nutritional content were identical in the two shakes.

\subsection{Instruments}

2.4.1. Fibromyalgia Impact Questionnaire. The FIQ is a validated questionnaire that was developed to measure status, progress and outcomes of people with FMS [14]. A selfadministered instrument, it takes $\sim 5 \mathrm{~min}$ to complete. The FIQ contains 20 items that measure physical functioning; symptoms of pain, fatigue, morning tiredness and stiffness; job difficulty; depression and anxiety; days of work missed; and overall well-being of the person during the previous week. A higher score indicates a greater effect of FMS on the person, with a range of total score from 0 to 100 .

Questions 1 through 11 rate the ability to complete various activities and are scored and summed to yield 1 physical impairment score ( 0 , no impairment; 10 , maximum impairment). Question 12 inquires about the number of days out of the past 7 when the patient felt good, and question 13 inquires about the number of days during the past week when the patient missed work, including housework, because of fibromyalgia, with each question yielding a separate score ( 0 , no impairment; 10 , maximum impairment). Questions 14 through 20 are a series of visual analog scales (range, 0 10) for rating the various symptoms characteristic of FMS ( 0 , no impairment; 70, maximum impairment).

2.4.2. Center for Epidemiologic Studies Depression Scale. The CES-D is a 20-item measure of depression. Its questions represent depressed mood; feelings of guilt, worthlessness, helplessness or hopelessness; psychomotor retardation; loss of appetite; and sleep disturbance [15]. Scale scores can range from 0 to 60; a higher number indicates greater depression. Various cutoff points for depression have been used. For example, Weissman et al. [16] used the cutoff of 16 and Turk et al. [17] the cutoff of 19 .

2.5. Statistical Analysis. Patient demographics were summarized using descriptive statistics. The mean $( \pm S D)$ of the total FIQ scores and the CES-D scores at baseline and at 6 weeks between the soy group and the placebo group were analyzed by Wilcoxon rank sum test. The difference and relative change (\%) of the FIQ and CES-D scores at 6 weeks from baseline were also compared by Wilcoxon rank sum test. The comparison of the difference from baseline to 6 weeks within the soy and placebo groups was analyzed by the Wilcoxon signed rank test. The analyses were performed using intent-to-treat and per-protocol approaches. In intentto-treat analysis, a participant who did not complete the entire 6-week supplement trial or who failed to complete the FIQ and CES-D forms was considered a dropout.

Because this study was an early phase trial with a small sample size, we also analyzed our data by using the separation test, as described by Aickin $[18,19]$, to assess whether it is worthwhile to pursue research on soy supplementation for patients with fibromyalgia. By use of this test, the standard deviation of the effect estimate (SDE) of the mean difference can be found. The value of $\Delta=1.645^{*} \mathrm{SDE}$ is then calculated. If the mean difference exceeds $\Delta / 2$ (in the favorable direction), further research is recommended; if it decreases below $-\Delta / 2$ (in the unfavorable direction), further research is not recommended. Otherwise, the statement is made that there is not enough information to make a recommendation.

\section{Results}

3.1. Baseline Characteristics. A total of 50 patients (49 women) were recruited for this trial. Median age was 47.7 years (range, $18-76$ years). There was no significant difference in age $(P=.99)$, FIQ scores $(P=.36)$ or CES$\mathrm{D}$ scores $(P=.48)$ between the two groups. Twenty-eight patients (56\%) -12 in the soy group and 16 in the placebo group $(P=.39)$-completed the 6-week trial. Reasons for not completing it are depicted in Figure 1. Patients who did not finish the trial were significantly younger (median age, 39.8 years) than those who finished it (median age, 53.9 years $)(P<.001)$. The median FIQ score was higher for patients who did not complete the trial (59.5) than for those who completed the study (54.8), but the difference was not statistically significant $(P=.12)$. The median CES-D score also was higher for patients who did not complete the trial (26.5) than for patients who completed the trial (14.0), but this also was not a statistically significant difference $(P=.14)$.

3.2. Between-Group Comparisons. With intent-to-treat analysis, the total FIQ scores determined at study entry and at study completion decreased $14 \%( \pm 29)$ in the soy group and $18 \%( \pm 25)$ in the placebo group $(P=.16)$. With per-protocol analysis, the total FIQ scores decreased $29 \%( \pm 36)$ in the soy group and $28 \%( \pm 26)$ in the placebo group $(P=.93)$ (Figure 2). No statistically significant decrease between the soy group and the placebo group was observed for any of the FIQ subclass scores. With intent-to-treat analysis, the CES-D scores improved $16 \%( \pm 26)$ in the soy group and $15 \%( \pm 41)$ in the placebo group $(P=.83)$; with per-protocol analysis, the CES-D scores improved 33\% $( \pm 30)$ in the soy group and $24 \%( \pm 50)$ in the placebo group (Figure 3$)$. However, the decrease between the groups was not statistically significant $(P=.96)$. Using various cutoff points for depression (CES$\mathrm{D} \geq 16$, CES-D $\geq 19$ and CES-D $\geq 27$ ) and intent-totreat analysis, we found a depression rate of $52 \%, 48 \%$ and $28 \%$, respectively, in the soy group and $52 \%, 48 \%$ and $32 \%$ in the control group at study entry. After 6 weeks, these percentages were $48 \%, 44 \%$ and $24 \%$ in the treatment group and $48 \%, 36 \%$ and $24 \%$ in the control group. There was no statistically significant difference between the groups $(P$ $=1.00$ ). Determined by using per-protocol analysis for these three different CES-D scores, the percentages of patients with depression at study entry in the treatment group were $42 \%$, $33 \%$ and $8 \%$ and in the control group were $38 \%, 38 \%$ and $19 \%$. After 6 weeks, $33 \%$ of the treatment group had CESD scores of 16 or greater, $25 \%$ had scores of 19 or greater and $0 \%$ had scores of 27 or greater. The percentages for the 


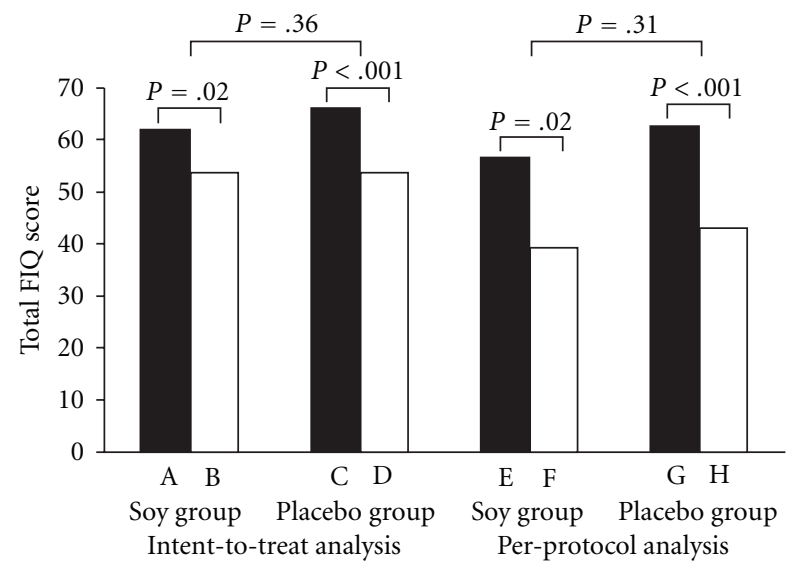

FIgUre 2: Total FIQ scores of patients in soy supplement trial, with intent-to-treat analysis (A-D) and per-protocol analysis (E$\mathrm{H})$. Score of patients randomly assigned to receive soy at study entry (A) and score of patients after 6 weeks of soy treatment (B). Score of patients randomly assigned to receive placebo at study entry (C) and score of patients after 6 weeks of placebo (D). Score of patients randomly assigned to receive soy at study entry (E) and score of patients after 6 weeks of soy treatment (F). Score of patients randomly assigned to receive placebo at study entry $(G)$ and score of patients after 6 weeks of placebo $(\mathrm{H})$.

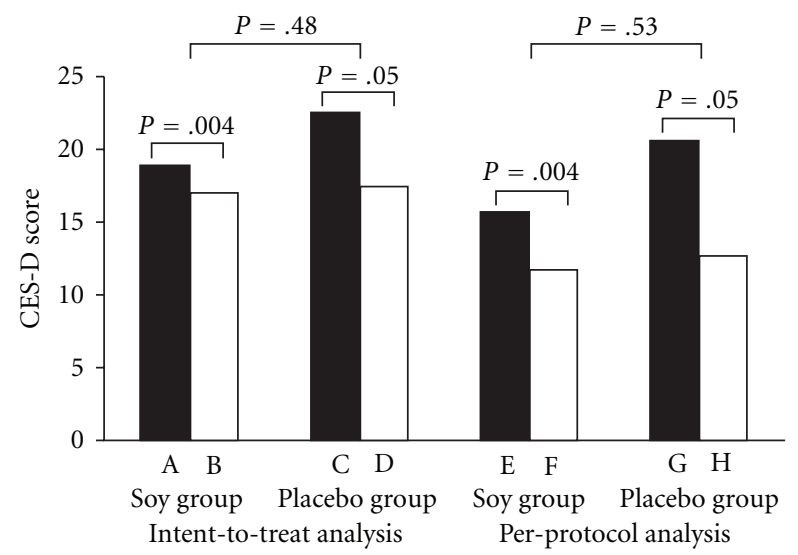

Figure 3: CES-D scores of patients in soy supplement trial, with intent-to-treat analysis (A-D) and per-protocol analysis (E-H). Score of patients randomly assigned to receive soy at study entry (A) and score of patients after 6 weeks of soy treatment (B). Score of patients randomly assigned to receive placebo at study entry (C) and score of patients after 6 weeks of placebo (D). Score of patients randomly assigned to receive soy at study entry (E) and score of patients after 6 weeks of soy treatment (F). Score of patients randomly assigned to receive placebo at study entry $(G)$ and score of patients after 6 weeks of placebo $(\mathrm{H})$.

control group were $31 \%, 19 \%$ and $6 \%$, respectively. There was no statistically significant difference between the groups.

The separation test using the difference from baseline to 6 weeks showed no benefit for soy, as determined by FIQ scores and CES-D scores for both intent-to-treat and perprotocol analyses. We did not have enough information to make any recommendation for or against the use of soy using the separation test per-protocol analysis based on total FIQ scores (Table 1).

3.3. Within-Group Comparisons. Significant, but modest, improvement in total FIQ scores (soy group, $P=.02$; placebo group, $P<.001$ ) (Figure 2 ) and CES-D scores (soy group, $P=$ .004 ; placebo group, $P=.05$ ) (Figure 3 ) between study entry and study completion was seen in both groups. Using three different cutoff points for depression (CES-D $\geq 16$, CES-D $\geq$ 19 and CES-D $\geq 27$ ), we found no significant improvement using the McNemar test for the soy and control groups $(P>$ $.50)$.

3.3.1. FIQ Subclass Scores. The average score of answers to questions 1 through 11 showed no significant improvement from before treatment to after treatment in both groups, as did the average score of answers to questions 12 and 13. However, the average score of answers to questions 14 through 20 showed significant improvement from before treatment to after treatment in the soy group $(P=.004)$ and the placebo group $(P=.001)$.

\section{Discussion}

The present study shows that use of soy product and use of the chosen placebo for 6 weeks, when combined with an educational intervention, were both associated with modest improvement in symptoms of fibromyalgia and depression.

Patients seen in the Mayo Fibromyalgia Treatment Program have moderate to severe symptoms, as demonstrated by the high total FIQ score of our sample at study entry (average score, $59.4 \pm 13.3$ ). A 1.5-day multidisciplinary treatment program, such as the program developed at our institution is considered standard care for these patients and has been shown to have a significant positive effect on the impact of illness among patients with FMS [13]. Despite participation in such multidisciplinary treatment programs and use of many conventional medications (e.g., tricyclic antidepressants, selective serotonin reuptake inhibitors, dual serotonin and norepinephrine reuptake inhibitors, analgesics, anticonvulsants), most patients with FMS continue to have functionally limiting symptoms [20].

Soy consumption has been reported in the CAM literature to have many beneficial effects on bone health, the cardiovascular system and degenerative arthritis and has been shown in epidemiologic studies to be associated with lower risk of several cancers [21]. Intrigued by animal studies indicating that dietary soy provided relief in neuropathic pain, we decided to perform this early phase trial [8-11]. Using standard statistics based on the null hypothesis, we show that the use of dietary soy is no more helpful than the use of casein when each is combined with a brief multidisciplinary treatment program. Although we cannot exclude the high drop-out rate, a patient population with severe symptoms as a result of referral bias, and a lack of efficacy of the soy product as reasons for these findings, another possible cause is the small sample size of our study, which represents an early phase trial. In concordance with 
TABLE 1: Summary of data analysis with a separation test ${ }^{\mathrm{a}}$.

\begin{tabular}{|c|c|c|c|c|c|c|c|c|c|}
\hline \multirow[t]{2}{*}{ Outcome } & \multicolumn{2}{|c|}{ Control (placebo) } & \multicolumn{2}{|c|}{ Treatment (soy) } & \multirow{2}{*}{ SDE } & \multirow{2}{*}{$\Delta / 2^{\mathrm{b}}$} & \multirow{2}{*}{$\begin{array}{l}\text { Mean } \\
\text { difference } \\
\text { (placebo/soy) }\end{array}$} & \multirow{2}{*}{$\begin{array}{l}\text { Separation } \\
\text { in favor of } \\
\text { placebo or } \\
\text { soy }^{c}\end{array}$} & \multirow{2}{*}{$\begin{array}{l}\text { Further } \\
\text { research } \\
\text { with soy } \\
\text { indicated? }\end{array}$} \\
\hline & $\begin{array}{l}\text { Mean } \\
\text { change in } \\
\text { scores }\end{array}$ & $95 \%$ CI & $\begin{array}{l}\text { Mean } \\
\text { change in } \\
\text { scores }\end{array}$ & $95 \%$ CI & & & & & \\
\hline \multicolumn{10}{|c|}{ Intent-to-treat analysis } \\
\hline $\mathrm{F} 1-11^{\mathrm{d}}$ & -0.51 & -3.55 to 2.54 & -0.07 & -1.93 to 1.79 & 0.18 & 0.15 & -0.44 & Placebo & No \\
\hline $\mathrm{F} 12^{\mathrm{d}}$ & -2.06 & -8.69 to 4.57 & -1.26 & -7.00 to 4.48 & 0.45 & 0.37 & -0.80 & Placebo & No \\
\hline $\mathrm{F} 13^{\mathrm{d}}$ & -1.12 & -7.01 to 4.77 & -0.32 & -5.08 to 4.44 & 0.39 & 0.32 & -0.80 & Placebo & No \\
\hline $\mathrm{F} 14-20^{\mathrm{d}}$ & -8.84 & -38.73 to 21.05 & -6.56 & -29.36 to 16.24 & 1.91 & 1.57 & -2.28 & Placebo & No \\
\hline Total FIQ scores & -12.52 & -53.34 to 28.29 & -8.21 & -39.39 to 22.97 & 2.61 & 2.15 & -4.32 & Placebo & No \\
\hline Total CES-D scores & -5.12 & -31.60 to 21.36 & -1.92 & -8.12 to 4.28 & 1.39 & 1.15 & -3.20 & Placebo & No \\
\hline \multicolumn{10}{|l|}{ Per-protocol analysis } \\
\hline $\mathrm{F} 1-11^{\mathrm{d}}$ & -0.79 & -4.52 to 2.94 & -0.15 & -2.89 to 2.59 & 0.32 & 0.27 & -0.64 & Placebo & No \\
\hline $\mathrm{F} 12^{\mathrm{d}}$ & -3.22 & -10.63 to 4.20 & -2.62 & -10.16 to 4.92 & 0.71 & 0.58 & -0.60 & Placebo & No \\
\hline $\mathrm{F} 13^{\mathrm{d}}$ & -1.75 & -8.89 to 5.39 & -0.67 & -7.63 to 6.29 & 0.68 & 0.56 & -1.08 & Placebo & No \\
\hline $\mathrm{F} 14-20^{\mathrm{d}}$ & -13.81 & -47.69 to 20.07 & -13.67 & -40.64 to 13.30 & 2.95 & 2.42 & -0.15 & Neither & $\ldots^{\mathrm{e}}$ \\
\hline Total FIQ scores & -19.57 & -65.40 to 26.26 & -17.10 & -55.62 to 21.42 & 4.06 & 3.34 & -2.47 & Neither & $\ldots$ \\
\hline Total CES-D scores & -8.00 & -40.05 to 24.05 & -4.00 & -10.99 to 2.99 & 2.37 & 1.95 & -4.00 & Placebo & No \\
\hline
\end{tabular}

Aickin [18, 19], we believe that early phase research in CAM is important because of its large influence on the subsequent expenditure of money and effort in CAM therapies. By analyzing our data with separation testing, we were unable to document any separation between soy use and casein use (control) through FIQ and CES-D scores in favor of soy when using intent-to-treat analysis, as well as per-protocol analysis. Our interpretation of these results is that further studies using large sample sizes of patients with fibromyalgia are unlikely to show a positive effect of soy supplement compared with placebo and therefore are not indicated.

Given the prevalence of CAM, the many various CAM therapies used for patients with FMS, and the cost associated with their use, it appears prudent that each of these therapies should be carefully assessed, initially with early phase trials. If the early phase trials result in promising findings, adequately powered clinical trials should be performed.

The results of this randomized, double-blind, placebocontrolled, early phase trial of soy shakes for patients with FMS suggests that, on the basis of FIQ scores and CES-D scores, dietary soy supplementation is no more beneficial than casein shakes.

\section{Acknowledgments}

The authors acknowledge the study sponsor, Physicians Pharmaceuticals, Inc, Kernersville, North Carolina, for its support of this study.

\section{References}

[1] D. L. Goldenberg, C. Burckhardt, and L. Crofford, "Management of fibromyalgia syndrome," Journal of the American Medical Association, vol. 292, no. 19, pp. 2388-2395, 2004.

[2] F. Wolfe, H. A. Smythe, M. B. Yunus et al., "The American College of Rheumatology 1990. Criteria for the classification of fibromyalgia. Report of the Multicenter Criteria Committee," Arthritis and Rheumatism, vol. 33, no. 2, pp. 160-172, 1990.

[3] L. C. Holdcraft, N. Assefi, and D. Buchwald, "Complementary and alternative medicine in fibromyalgia and related syndromes," Best Practice and Research: Clinical Rheumatology, vol. 17, no. 4, pp. 667-683, 2003.

[4] J. K. Rao, K. Mihaliak, K. Kroenke, J. Bradley, W. M. Tierney, and M. Weinberger, "Use of complementary therapies for arthritis among patients of rheumatologists," Annals of Internal Medicine, vol. 131, no. 6, pp. 409-416, 1999.

[5] C. J. Herman, P. Allen, W. C. Hunt, A. Prasad, and T. J. Brady, "Use of complementary therapies among primary care clinic patients with arthritis," Preventing Chronic Disease, vol. 1, no. 4, p. A12, 2004.

[6] D. L. Wahner-Roedler, P. L. Elkin, A. Vincent et al., "Use of complementary and alternative medical therapies by patients referred to a fibromyalgia treatment program at a Tertiary Care Center," Mayo Clinic Proceedings, vol. 80, no. 1, pp. 5560, 2005.

[7] E. Ernst, "Musculoskeletal conditions and complementary/alternative medicine," Best Practice and Research: Clinical Rheumatology, vol. 18, no. 4, pp. 539-556, 2004.

[8] Y. Shir, S. N. Raja, C. S. Weissman, J. N. Campbell, and Z. Seltzer, "Consumption of soy diet before nerve injury 
preempts the development of neuropathic pain in rats," Anesthesiology, vol. 95, no. 5, pp. 1238-1244, 2001.

[9] Y. Shir, R. Sheth, J. N. Campbell, S. N. Raja, and Z. Seltzer, "Soy-containing diet suppresses chronic neuropathic sensory disorders in rats," Anesthesia and Analgesia, vol. 92, no. 4, pp. 1029-1034, 2001.

[10] Y. Shir, J. N. Campbell, S. N. Raja, and Z. Seltzer, "The correlation between dietary soy phytoestrogens and neuropathic pain behavior in rats after partial denervation," Anesthesia and Analgesia, vol. 94, no. 2, pp. 421-426, 2002.

[11] J. Pérez, M. A. Ware, S. Chevalier, R. Gougeon, G. J. Bennett, and Y. Shir, "Dietary fat and protein interact in suppressing neuropathic pain-related disorders following a partial sciatic ligation injury in rats," Pain, vol. 111, no. 3, pp. 297-305, 2004.

[12] B. H. Arjmandi, D. A. Khalil, E. A. Lucas et al., "Soy protein may alleviate osteoarthritis symptoms," Phytomedicine, vol. 11, no. 7-8, pp. 567-575, 2004.

[13] A. Pfeiffer, J. M. Thompson, A. Nelson et al., "Effects of a 1.5-day multidisciplinary outpatient treatment program for fibromyalgia: a pilot study," American Journal of Physical Medicine and Rehabilitation, vol. 82, no. 3, pp. 186-191, 2003.

[14] C. S. Burckhardt, S. R. Clark, and R. M. Bennett, "The fibromyalgia impact questionnaire: development and validation," Journal of Rheumatology, vol. 18, no. 5, pp. 728-733, 1991.

[15] L. S. Radloff, "The CES-D Scale: a self-report depression scale for research in the general population," Applied Psychological Measurement, vol. 1, pp. 385-401, 1977.

[16] M. M. Weissman, J. K. Myers, and P. S. Harding, "Psychiatric disorders in a U.S. urban community: 1975-1976," American Journal of Psychiatry, vol. 135, no. 4, pp. 459-462, 1978.

[17] D. C. Turk, A. Okifuji, J. D. Sinclair, and T. W. Starz, "Interdisciplinary treatment for fibromyalgia syndrome: clinical and statistical significance," Arthritis Care and Research, vol. 11, no. 3, pp. 186-195, 1998.

[18] M. Aickin, "Separation tests for early-phase complementary and alternative medicine comparative trials," Evidence-Based Integrative Medicine, vol. 1, no. 4, pp. 225-232, 2004.

[19] M. Aickin, “The importance of early phase research," Journal of Alternative and Complementary Medicine, vol. 13, no. 4, pp. 447-450, 2007.

[20] F. Wolfe, J. Anderson, D. Harkness et al., "Health status and disease severity in fibromyalgia: results of a six-center longitudinal study," Arthritis and Rheumatism, vol. 40, no. 9, pp. 1571-1579, 1997.

[21] P. S. Haddad, G. A. Azar, S. Groom, and M. Boivin, "Natural health products, modulation of immune function and prevention of chronic diseases," Evidence-Based Complementary and Alternative Medicine, vol. 2, no. 4, pp. 513-520, 2005. 


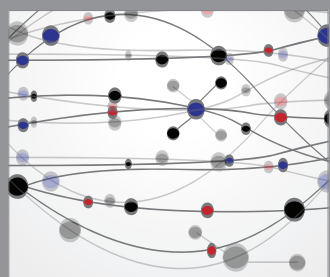

The Scientific World Journal
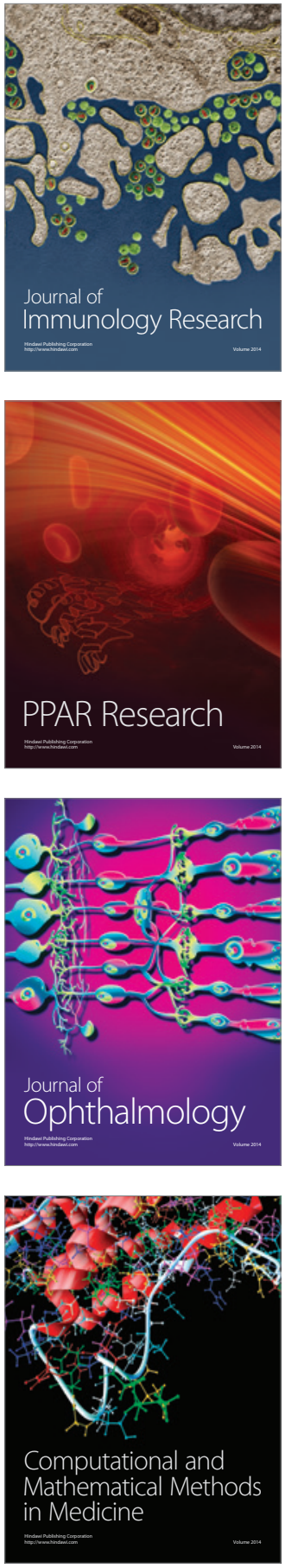

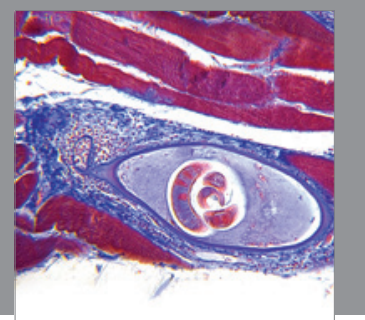

Gastroenterology

Research and Practice
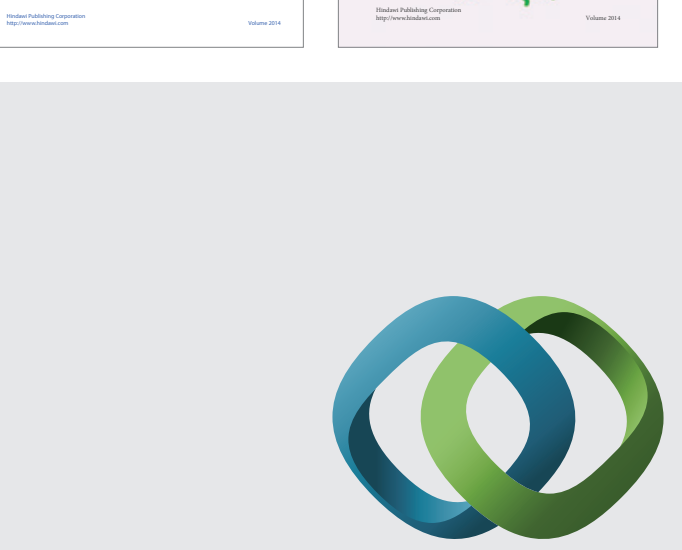

\section{Hindawi}

Submit your manuscripts at

http://www.hindawi.com
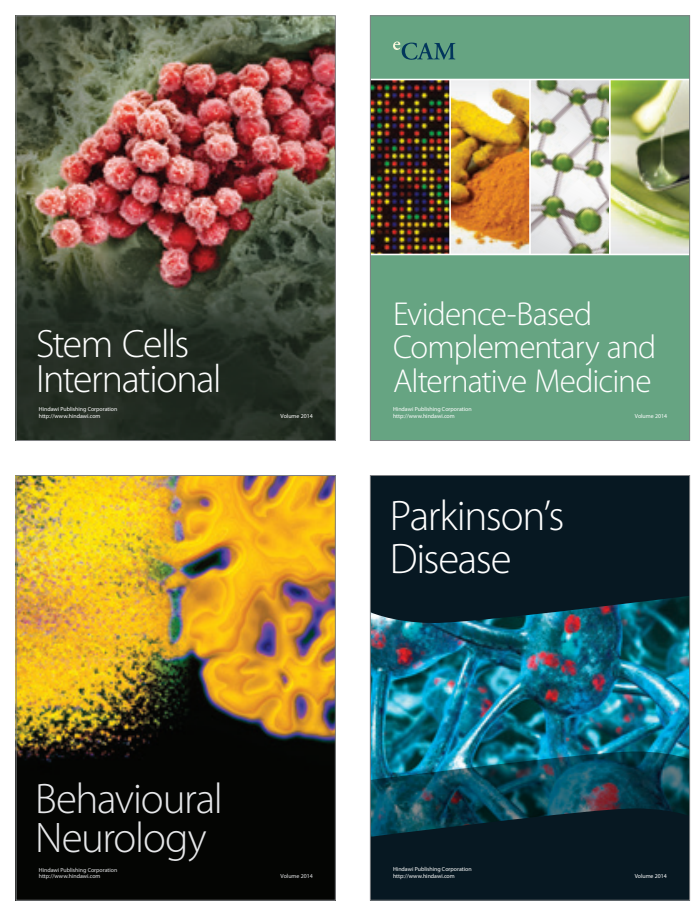

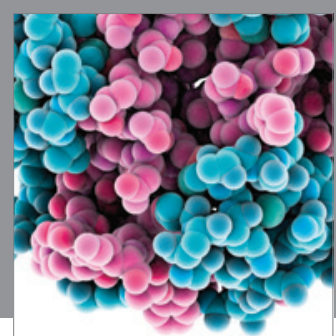

Journal of
Diabetes Research

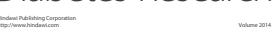

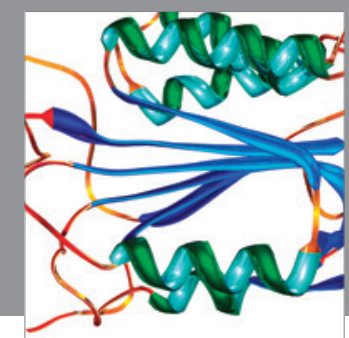

Disease Markers
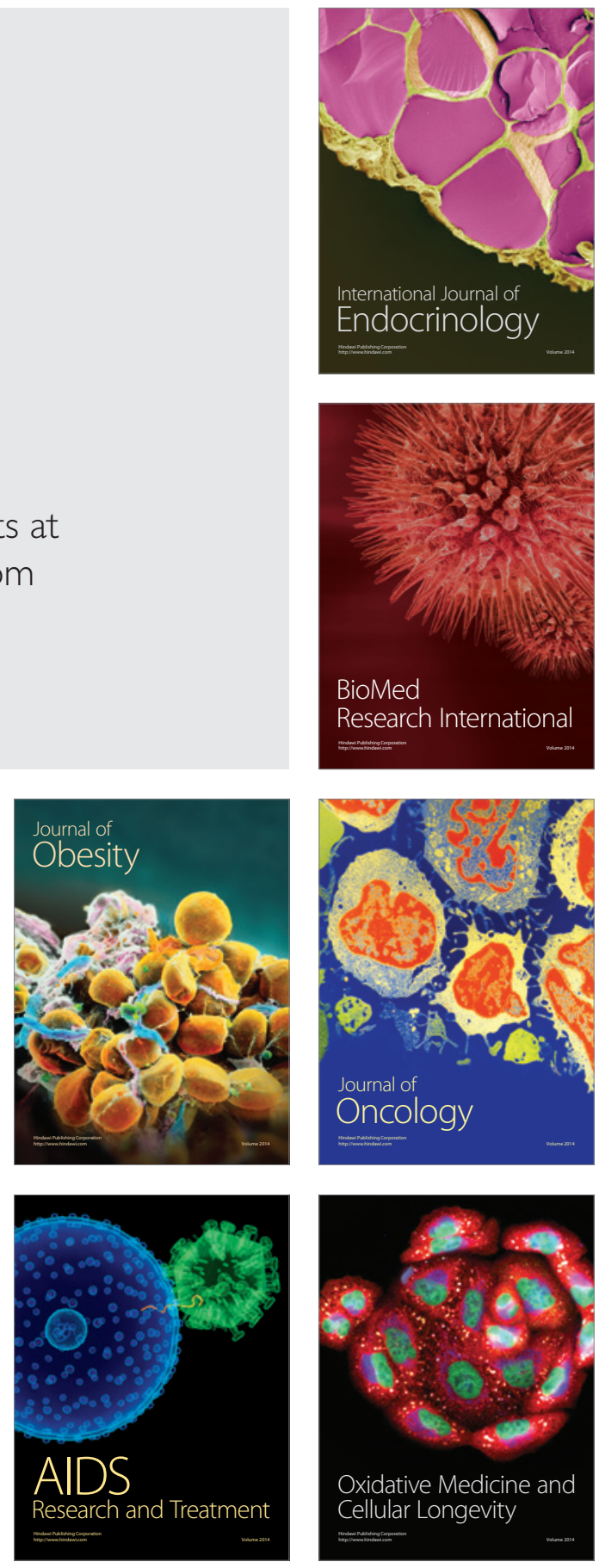